\title{
The influence of a micropolar fluid on peristaltic transport in an annulus: application of the clot model
}

\author{
Kh. S. Mekheimer ${ }^{\mathrm{a} *}$ and Y. Abd Elmaboud ${ }^{\mathrm{b}}$ \\ ${ }^{a}$ Mathematics Department, Faculty of Science, Al-Azhar University, Nasr City, 11884 Cairo, Egypt; \\ ${ }^{b}$ Mathematics Department, Faculty of Science, Al-Azhar University (Assiut Branch), Assiut, Egypt
}

(Received 14 February 2008; final version received 8 June 2008)

\begin{abstract}
A serious pathological condition is encountered when some blood constituents deposited on the blood vessels get detached from the wall, join the blood stream again and form a clot. Study of the peristaltic transport of a micropolar fluid in an annular region is investigated under low Reynolds number and long wavelength approximations. We model a small artery as a tube having a sinusoidal wave travelling down its wall and a clot model inside it. Closed form solutions are obtained for the velocity and the microrotation components, as well as the stream function, and they contain new additional parameters, namely, $\delta$, the height of the clot, $N$, the coupling number and $m$, the micropolar parameter. The pressure rise and friction force on the inner and the outer tubes have been discussed for various values of the physical parameters of interest.
\end{abstract}

Keywords: peristaltic transport; micropolar fluid; clot model; pressure rise

\section{Introduction}

Peristaltic pumping is a form of fluid transport that occurs when a progressive wave of area contraction or expansion propagates along the length of a distensible duct. Peristalsis is an inherent property of many biological systems having smooth muscle tubes that transports biofluids by its propulsive movement and is found in the transport of urine from kidney to the bladder, the movement of chyme in the gastrointestinal tract, intra-uterine fluid motion, vasomotion of the small blood vessels and in many other glandular ducts. The mechanism of peristaltic transport has been exploited for industrial applications such as sanitary fluid transport, blood pumps in heart-lung machine and transport of corrosive fluids where the contact of the fluid with the machinery parts is prohibited. The behaviour of most of the physiological fluids is known to be non-Newtonian. Hence, in recent years, the study of peristaltic transport of non-Newtonian fluids in channels or pipes has gained much attention. Several investigators, Provost and Schwarz (1994), Srivastava and Srivastava (1988), Vajravelu et al. (2005), Rao and Mishra (2004) and Hayat et al. (2005), have analysed the peristaltic transport in physiological situations of interest. Most of these analytical studies use asymptotic expansions with small Reynolds number, wave number, and amplitude ratio as the perturbation parameters. The model of micropolar fluid introduced by Eringen (1966) represents fluids consisting of rigid, randomly oriented (or spherical) particles suspended in a viscous medium where the deformation of the particles is ignored. Micropolar fluids exhibit some microscopic effects arising from the local structure and micromotion of the fluid elements. Furthermore, they can sustain couple stresses. The micropolar fluid is considered to model the blood flow in small arteries and the calculation of theoretical velocity profiles is observed in good agreement with the experimental data. A serious pathological condition is encountered when some blood constituents deposited on the artery wall get detached from the wall, join the blood stream again and form a clot. This can lead to partial or even complete blockage of the blood vessels. Jayaraman and Sarkar (2005) studied the mathematical model formulated for solving flow in a stenosed artery with a clot inside it. In the present study, the peristaltic transport of an incompressible micropolar fluid in an annular region between two coaxial tubes, the outer tube is uniform and has a sinusoidal wave travelling down its wall and the inner one has a clot on its wall, is investigated. The analysis has been carried out in the wave frame of reference with long wavelength and zero Reynolds number assumptions. The relationship between pressure gradient and time mean flow rate for various micropolar parameters, coupling numbers and maximum height attained by the clot is obtained. A motivation of the present analysis is the hope that such a problem will be applicable in many clinical applications.

\section{Formulation of the problem}

Consider the flow of an incompressible micropolar fluid in an annular region between two coaxial tubes, the outer tube is uniform and has a sinusoidal wave travelling down

*Corresponding author. Email: kh_mekheimer@yahoo.com

ISSN: $1176-2322$ print / 1754-2103 online

Copyright (C) 2008 Taylor \& Francis

DOI: $10.1080 / 11762320802256666$

http://www.informaworld.com 


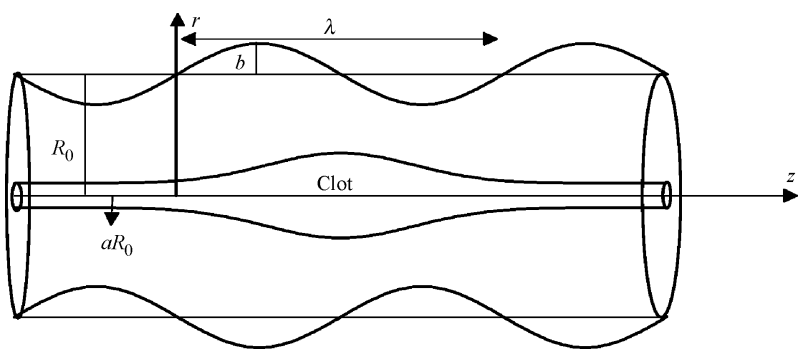

Figure 1. Geometry of the problem.

its wall and the inner one having a clot on its wall. The geometries of the wall surfaces are (see Figure 1).

$$
\begin{array}{rlr}
R_{1}^{\prime} & =R_{0}\left(a+f\left(Z^{\prime}, t^{\prime}\right)\right), \quad 0 \leq Z^{\prime} \leq \lambda, \\
& =R_{0} a \quad \text { otherwise, } \\
R_{2}^{\prime} & =R_{0}+b \sin \left(\frac{2 \pi}{\lambda}\left(Z^{\prime}-c t^{\prime}\right)\right),
\end{array}
$$

where $a R_{0}$ is the radius of the inner tube that keeps the clot model in position inside the tube with $a \ll 1, f\left(Z^{\prime}, t^{\prime}\right)$ the arbitrary shape along the axial direction that can be handled through suitable choice, $R_{0}$ is the radius of the outer tube at any axial distance $Z^{\prime}$ from the inlet, $b$ is the wave amplitude, $\lambda$ is the wavelength, $c$ is the propagation velocity and $t^{\prime}$ is the time. Introducing a wave frame $\left(r^{\prime}, z^{\prime}\right)$ moving with velocity $c$ away from the fixed frame $\left(R^{\prime}, Z^{\prime}\right)$ by the transformation

$$
z^{\prime}=Z^{\prime}-c t, \quad r^{\prime}=R^{\prime}, \quad v_{z}^{\prime}=V_{z}^{\prime}-c, \quad v_{r}^{\prime}=V_{r}^{\prime},
$$

where $\left(V_{r}^{\prime}, V_{z}^{\prime}\right)$ and $\left(v_{r}^{\prime}, v_{z}^{\prime}\right)$ are radial and axial velocity components in the stationary and moving coordinate systems, respectively.

Taking the axisymmetric flow, where all the variables are independent of $\theta$, the velocity vector is given by $\mathbf{v}^{\prime}=$ $\left(v_{r}^{\prime}, 0, v_{z}^{\prime}\right)$ and microrotation vector is $\mathbf{w}^{\prime}=\left(0, v_{\theta}^{\prime}, 0\right)$. Also, we introduce the following non-dimensional variables:

$$
\begin{aligned}
& r=\frac{r^{\prime}}{R_{0}}, z=\frac{z^{\prime}}{\lambda}, v_{z}=\frac{v_{z}^{\prime}}{c}, v_{r}=\frac{\lambda}{c R_{0}} v_{r}^{\prime}, v_{\theta}=\frac{R_{0}}{c} v_{\theta}^{\prime}, \\
& p=\frac{R_{0}^{2}}{\lambda \mu c} p^{\prime}, t=\frac{c}{\lambda} t^{\prime}, j=\frac{j^{\prime}}{R_{0}^{2}}, r_{1}=\frac{r_{1}^{\prime}}{R_{0}}, r_{2}=\frac{r_{2}^{\prime}}{R_{0}}
\end{aligned}
$$

After using these transformation and non-dimensional variables, the non-dimensional equations governing the steady flow of an incompressible micropolar fluid in the absence of body force and body couple are:

$$
\begin{aligned}
& \frac{\partial v_{r}}{\partial r}+\frac{\partial v_{z}}{\partial z}+\frac{v_{r}}{r}=0, \\
& \operatorname{Re} \alpha^{3}\left(v_{r} \frac{\partial v_{r}}{\partial r}+v_{z} \frac{\partial v_{r}}{\partial z}\right)=-\frac{\partial p}{\partial r}+\frac{\alpha^{2}}{1-N}
\end{aligned}
$$

$$
\begin{gathered}
\times\left(-N \frac{\partial v_{\theta}}{\partial z}+\frac{\partial^{2} v_{r}}{\partial r^{2}}+\frac{1}{r} \frac{\partial v_{r}}{\partial r}-\frac{v_{r}}{r^{2}}+\alpha^{2} \frac{\partial^{2} v_{r}}{\partial z^{2}}\right), \\
\operatorname{Re} \alpha\left(v_{r} \frac{\partial v_{z}}{\partial r}+v_{z} \frac{\partial v_{z}}{\partial z}\right)=-\frac{\partial p}{\partial z}+\frac{1}{1-N} \\
\times\left(\frac{N}{r} \frac{\partial\left(r v_{\theta}\right)}{\partial r}+\frac{\partial^{2} v_{z}}{\partial r^{2}}+\frac{1}{r} \frac{\partial v_{z}}{\partial r}+\alpha^{2} \frac{\partial^{2} v_{z}}{\partial z^{2}}\right), \\
\frac{j \operatorname{Re} \alpha(1-N)}{N}\left(v_{r} \frac{\partial v_{\theta}}{\partial r}+v_{z} \frac{\partial v_{\theta}}{\partial z}\right)=-2 v_{\theta}+\left(\alpha^{2} \frac{\partial v_{r}}{\partial z}-\frac{\partial v_{z}}{\partial r}\right) \\
+\frac{2-N}{m^{2}}\left(\frac{\partial}{\partial r}\left(\frac{1}{r} \frac{\partial\left(r v_{\theta}\right)}{\partial r}\right)+\alpha^{2} \frac{\partial^{2} v_{\theta}}{\partial z^{2}}\right),
\end{gathered}
$$

where $\phi=b / R_{0}$ is the amplitude ratio, $\alpha=R_{0} / \lambda, R e=$ $\rho c R_{0} / \mu$ is the Reynolds number, $N=k /(\mu+k)$ is the coupling number $(0 \leq N \leq 1)$ (Cowin 1968) and $m^{2}=R_{0}^{2} k(2 \mu+k) /(\gamma(\mu+k))$ is the micropolar parameter (Eringer 1966). Using the long wavelength approximation $\alpha \ll 1$ and dropping terms of order $\alpha$ and higher, it follows from Equations (5)-(8) that the appropriate equations describing the flow in the wave frame are:

$$
\begin{aligned}
\frac{\partial v_{r}}{\partial r}+\frac{\partial v_{z}}{\partial z}+\frac{v_{r}}{r} & =0 \\
\frac{\partial p}{\partial r} & =0 \\
\frac{N}{r} \frac{\partial\left(r v_{\theta}\right)}{\partial r} & +\frac{\partial^{2} v_{z}}{\partial r^{2}}+\frac{1}{r} \frac{\partial v_{z}}{\partial r}=(1-N) \frac{\partial p}{\partial z}, \\
2 v_{\theta} & +\frac{\partial v_{z}}{\partial r}-\frac{2-N}{m^{2}} \frac{\partial}{\partial r}\left(\frac{1}{r} \frac{\partial\left(r v_{\theta}\right)}{\partial r}\right)=0 .
\end{aligned}
$$

The corresponding boundary conditions in the wave frame are

$$
v_{z}=-1, \quad v_{\theta}=0 \quad \text { at } \quad r=r_{1}, \quad r=r_{2},
$$

where

$$
\begin{array}{rrr}
r_{1} & =a+f_{1}(z), \quad 0 \leq z \leq 1, \\
& =a \quad \text { otherwise, } \\
r_{2} & =1+\phi \sin (2 \pi z) .
\end{array}
$$

\section{Solution of the problem}

From Equations (10) and (11) and after one integration, we get

$$
\frac{\partial v_{z}}{\partial r}=(1-N)\left(\frac{r}{2} \frac{d p}{d z}+\frac{A_{1}(z)}{r}\right)-N v_{\theta} .
$$


On substituting Equation (16) into Equation (12), thus

$$
\begin{aligned}
\frac{\partial^{2} v_{\theta}}{\partial r^{2}} & +\frac{1}{r} \frac{\partial v_{\theta}}{\partial r}-\left(m^{2}+\frac{1}{r^{2}}\right) v_{\theta} \\
& =\frac{m^{2}(1-N)}{2-N}\left[\frac{r}{2} \frac{d p}{d z}+\frac{A_{1}(z)}{r}\right],
\end{aligned}
$$

and its general solution is

$$
\begin{aligned}
v_{\theta} & =A_{2}(z) I_{1}(m r)+A_{3}(z) K_{1}(m r) \\
& -\frac{(1-N)}{2-N}\left[\frac{r}{2} \frac{d p}{d z}+\frac{A_{1}(z)}{r}\right],
\end{aligned}
$$

where $I_{1}$ and $K_{1}$ are modified Bessel functions of the first order, first and second kinds, respectively. Substituting Equation (18) into Equation (16) and integrating, we obtain:

$$
\begin{aligned}
v_{z} & =\frac{N}{m}\left[A_{3}(z) K_{0}(m r)-A_{2}(z) I_{0}(m r)\right] \\
& +\frac{(1-N)}{2-N}\left(\frac{r^{2}}{2} \frac{d p}{d z}+2 A_{1}(z) \ln r\right)+A_{4}(z),
\end{aligned}
$$

where $I_{0}$ and $K_{0}$ are modified Bessel functions of the zeroth order. The expressions for $v_{z}$ and $v_{\theta}$ subject to the boundary conditions (14-15) are respectively given as

$$
\begin{aligned}
v_{z}= & \frac{1}{a_{24}}\left\{a_{39}+A F \frac{d p}{d z}\left(a_{48}\left(r^{2}-r_{2}^{2}\right)+a_{49}\left(r^{2}-r_{1}^{2}\right)\right.\right. \\
& \left.\left.+a_{41} K_{0}(m r)+a_{33} I_{0}(m r)+2 a_{32} r_{1} r_{2} \ln r+a_{34}\right)\right\} \\
v_{\theta}= & \frac{A}{2 r a_{53}} \frac{d p}{d z}\left\{F\left(r_{1}^{2}+r_{2}^{2}-2 r^{2}\right)+m r_{2} I_{1}\left(m r_{2}\right)\right. \\
& \times\left[F k_{0}\left(m r_{1}\right)\left(r^{2}-r_{1}^{2}\right)+a_{16}\left(r_{1} K_{1}\left(m r_{1}\right)-r K_{1}(m r)\right)\right. \\
& \left.+2 r r_{1} \ln \left(\frac{r_{2}}{r_{1}}\right)\left(r_{1} K_{1}(m r)-r K_{1}\left(m r_{1}\right)\right)\right] \\
& +a_{43} m r I_{1}(m r)+m\left(F \left[a_{44}\left(r^{2}-r_{1}^{2}\right)\right.\right. \\
& \left.+a_{13} a_{16} r K_{1}(m r)+a_{45}\left(r^{2}-r_{2}^{2}\right)\right]+a_{46}\left(r^{2}-r_{2}^{2}\right) \\
& \left.\left.+a_{54} r K_{1}(m r)-a_{47} K_{1}\left(m r_{2}\right)-a_{55}\right)\right\} .
\end{aligned}
$$

The corresponding stream function $\left(v_{r}=-\frac{1}{r} \frac{\partial \psi}{\partial z}\right.$ and $v_{z}=$ $\left.\frac{1}{r} \frac{\partial \psi}{\partial r}\right)$ is

$$
\begin{aligned}
\psi(r, z)= & b_{11}\left(r^{2}-r_{1}^{2}\right)+\frac{1}{4 m a_{24}}\left\{A F \frac{d p}{d z}\right. \\
& \left(m r^{2}\left(2 a_{34}-2 b_{13}+b_{14} r^{2}\right)-m b_{12} r_{1}^{2}-4 b_{15} r_{1}\right. \\
& +r\left(4\left(a_{33} I_{1}(m r)-a_{41} K_{1}(m r)\right)\right.
\end{aligned}
$$

$$
\begin{aligned}
& +m r\left[a _ { 3 5 } \left(\ln r_{1}\left(r^{2}-2 r_{2}^{2}\right)\right.\right. \\
& \left.\left.\left.\left.\left.+\ln r_{2}\left(r^{2}-2 r_{1}^{2}\right)\right)+4 a_{32} r_{1} r_{2} \ln r\right]\right)\right)\right\} .
\end{aligned}
$$

The dimensionless flux $\left(q=q^{\prime} / \pi c R_{0}^{2}, q^{\prime}\right.$ being the flux in the wave frame) is given by

$$
q=2 \int_{r_{1}}^{r_{2}} r v_{z} d r=\frac{1}{b_{19}}\left\{\eta \frac{d p}{d z}+\xi\right\},
$$

where the constants in Equations (20)-(23) are as given in Appendix 1. Thus, the pressure gradient is obtained from Equation (11) as

$$
\frac{d p}{d z}=\frac{1}{\eta}\left\{b_{19} q-\xi\right\} .
$$

Following the analysis given by Shapiro et al. (1969) the mean volume flow, $\bar{Q}$, over a period is obtained as

$$
\bar{Q}=\frac{1}{T} \int_{0}^{T}\left(q+\left(r_{2}^{2}-r_{1}^{2}\right)\right) d t=q+q_{2}-q_{1},
$$

where $q_{2}=\int_{0}^{1} r_{2}^{2} d z, q_{1}=\int_{0}^{1} r_{1}^{2} d z$. Substituting Equation (30) into Equation (29), we get

$$
\frac{d p}{d z}=\frac{1}{\eta}\left\{\left(\bar{Q}-q_{2}+q_{1}\right) b_{19}-\xi\right\} .
$$

The pressure rise $\Delta p$ and the friction force (at the wall) on the outer and inner tubes are $F^{(o)}$ and $F^{(i)}$, respectively, in their non-dimensional forms (Srivastava 1986).

$$
\begin{aligned}
\Delta p & =\int_{0}^{1} \frac{d p}{d z} d z, \quad F^{(o)}=\int_{0}^{1} r_{2}^{2}\left(-\frac{d p}{d z}\right) d z, \\
F^{(i)} & =\int_{0}^{1} r_{1}^{2}\left(-\frac{d p}{d z}\right) d z .
\end{aligned}
$$

In the absence of the inner tube (the clot model) (i.e. $r_{1} \rightarrow 0$ ), Equations (25)-(27) are

$$
\begin{aligned}
v_{z}= & -1+\frac{1-N}{2(2-N)} \frac{d p}{d z} \\
& {\left[r^{2}-r_{2}^{2}+\frac{N r_{2}}{m}\left(\frac{I_{0}\left(m r_{2}\right)-I_{0}(m r)}{I_{1}\left(m r_{2}\right)}\right)\right], } \\
v_{\theta}= & \frac{1-N}{2(2-N)} \frac{d p}{d z}\left[\frac{r_{2} I_{1}(m r)}{I_{1}\left(m r_{2}\right)}-r\right], \\
\psi(r, z)= & -\frac{r^{2}}{2}+\frac{1-N}{2(2-N)} \frac{d p}{d z}\left[\frac{r^{4}}{4}-\frac{r^{2} r_{2}^{2}}{2}+\frac{N r_{2}}{m I_{1}\left(m r_{2}\right)}\right. \\
& \left.\times\left(\frac{m r^{2} I_{0}\left(m r_{2}\right)-2 r I_{1}(m r)}{2 m}\right)\right] .
\end{aligned}
$$


These results are the same as those obtained by Srinivasacharya et al. (2003). Also, if we take $r_{1} \rightarrow 0$ and $k \rightarrow 0$ (i.e. $N \rightarrow 0$ ), the results coincide with that given by Shapiro et al. (1969) Substituting Equation (31) into Equations (32)(34), we get

$$
\begin{aligned}
\Delta p & =\int_{0}^{1} \frac{1}{\eta}\left\{\left(\bar{Q}-q_{2}+q_{1}\right) b_{19}-\xi\right\} d z, \\
F^{(o)} & =\int_{0}^{1}-\frac{r_{2}^{2}}{\eta}\left\{\left(\bar{Q}-q_{2}+q_{1}\right) b_{19}-\xi\right\} d z, \\
F^{(i)} & =\int_{0}^{1}-\frac{r_{1}^{2}}{\eta}\left\{\left(\bar{Q}-q_{2}+q_{1}\right) b_{19}-\xi\right\} d z,
\end{aligned}
$$

The integrals involved in Equations (38)-(40) are very difficult to evaluate analytically and thus are evaluated numerically.

\section{Numerical results and discussion}

The solutions are obtained for suitable choice of the clot model in the non-dimensional form (Jayaraman and Sarkar 2005) as

$$
r_{1}=a+\delta e^{-\pi^{2}\left(z-z_{d}-0.5\right)^{2}}, \quad 0 \leq z \leq 1
$$

where $\delta$ is the maximum height attained by the clot at $z=z_{d}+0.5, a$ is radius ratio of the inner tube that keeps the clot in position and $z_{d}$ represents the axial displacement of the clot. With the help of the MATHEMATICA program, the numerical evaluations of the analytical results are obtained for $\Delta p, F^{(o)}$ and $F^{(i)}$ for different parameters values (Eringen 1966; Jayaraman and Sarkar 2005; Srivastava 1986; Srinivascharya et al. 2003). The effect of the maximum height of the clot, $\delta$, is analysed by varying $\delta=0.05$ to $0.25, a=0.01,0.02, z_{d}=0,0.125,0 \leq N \leq 1$ and $m=0$ up to 100 . In Figure 2 the variation of $\Delta p$ with $\bar{Q}$ is shown for different values of $N$ and $\delta$ by fixing the other parameters, $a=0.02, \phi=0.4, z_{d}=0$ and $m=2$. It is noticed that a linear relation between them and an increase in the flow rate reduce the pressure rise and thus maximum flow rate is achieved at zero pressure rise and maximum pressure occurs at zero flow rate. The pressure rise increases as the coupling number $N$ increases, and $N=0$ corresponds to the case of Newtonian fluid. Also it is easy to observe that the pressure rise increases when the maximum height attained by the clot $\delta$ increases. Figure 3 depicts the variation of $\Delta p$ with $\bar{Q}$, with $N=0.4, z_{d}=0, \delta=0.1$ and $a=0.02$ for different values of micropolar parameter $m$ and the amplitude ratio $\phi$. An interesting observation here is that the pressure rise decreases with increases $m$ and is greater than the case of a Newtonian fluid $(m=0)$, but it increases with increases $\phi$. In Figure 4 the variation of $\Delta p$ with $\bar{Q}$ is shown for different values of $z_{d}$ and $a$, and fixing the other parameters, it is clear that the effect of increase $z_{d}$ and $a$

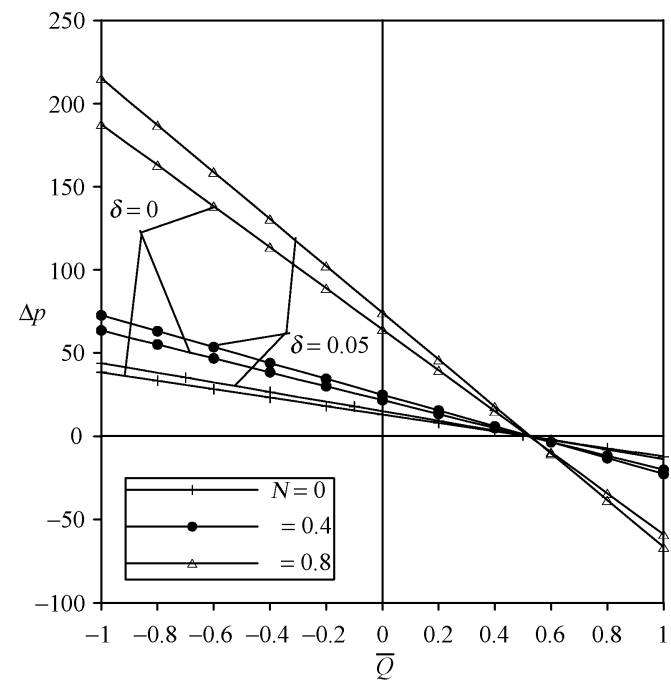

Figure 2. The variation of $\Delta p$ with $\bar{Q}$ for different values of $N$ and $\delta$ at $a=0.02, \phi=0.4, z_{d}=0, m=2$.

leads to increase $\Delta p$. Also Figures $2-4$ are divided into sectors to illustrate the pumping regions, peristaltic pumping $(\bar{Q}>0$ and $\Delta p>0)$, augmented pumping $(\bar{Q}>0$ and $\Delta p<0)$ and retrograde pumping $(\bar{Q}<0$ and $\Delta p>0)$ and it is clear that the peristaltic pumping region becomes wider with increases in $N, \delta$, amplitude ratio $\phi$ and $z_{d}$. The variation of $\Delta p$ with amplitude ratio $\phi$ for different values flow rate $\bar{Q}$ and the axial displacement of the clot $z_{d}$ for other given fixed set of parameters is presented in Figure 5. It is observed that the relation between $\Delta p$ and $\phi$ is a non-linear relation: $\Delta p$ increases with increases $\phi$. It is also evident that an increase in the flow rate reduces the pressure rise but an increase in the axial displacement of the clot $z_{d}$ increases the pressure rise. Figure 6 depicts the variation of $\Delta p$ with $\phi$ for different values of $N$ and $m$ for other given fixed set of

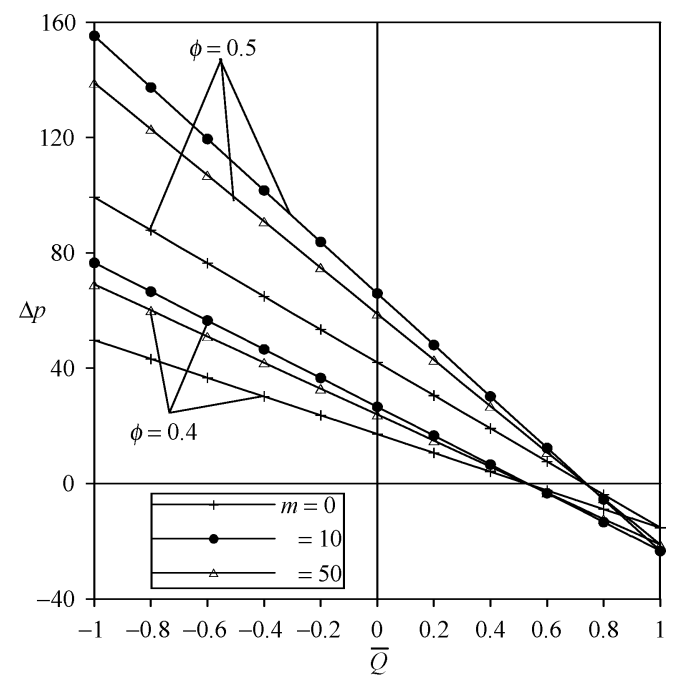

Figure 3. The variation of $\Delta p$ with $\bar{Q}$ for different values of $m$ and $\phi$ at $a=0.02, N=0.4, z_{d}=0, \delta=0.1$. 


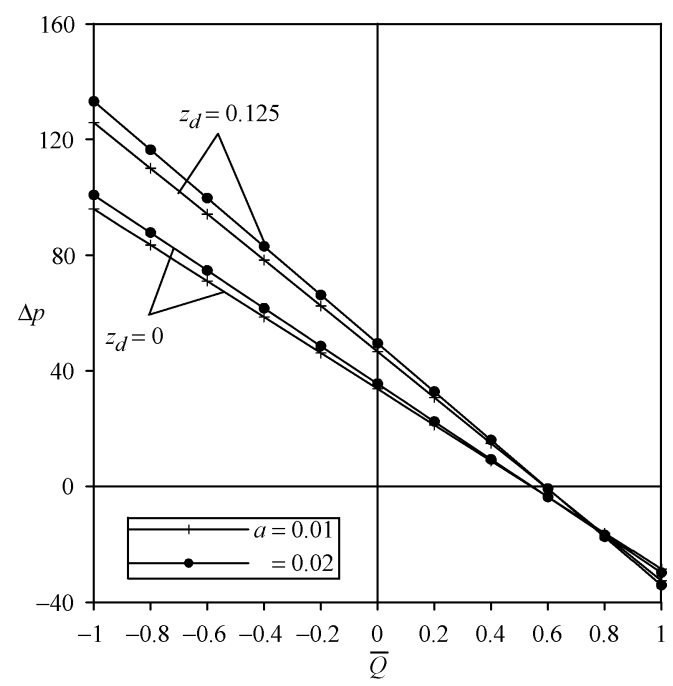

Figure 4. The variation of $\Delta p$ with $\bar{Q}$ for different values of $a$ and $z_{d}$ at $m=10, N=0.4, \phi=00.4, \delta=0.2$.

parameters. We observe that the pressure rise $\Delta p$ increases with increased $N$ and decreased $m$. Figures 7-9 describe the results obtained for the outer friction force versus the flow rate $\bar{Q}$, Figures 10 and 11 describe the results obtained for the outer friction force versus $\phi$, Figures 12-14 describe the results for inner friction force versus the flow rate $\bar{Q}$ and Figures 15 and 16 describe the results obtained for the inner friction force versus $\phi$. Furthermore, the effect of important parameters such as $N, m, \delta, a$ and $\phi$ on the inner and outer friction forces has been investigated. We notice from these figures that the inner and outer friction forces have the opposite behaviour compared to the pressure rise. The inner friction force behaves similar to the outer friction force for the same values of the parameters; moreover, the

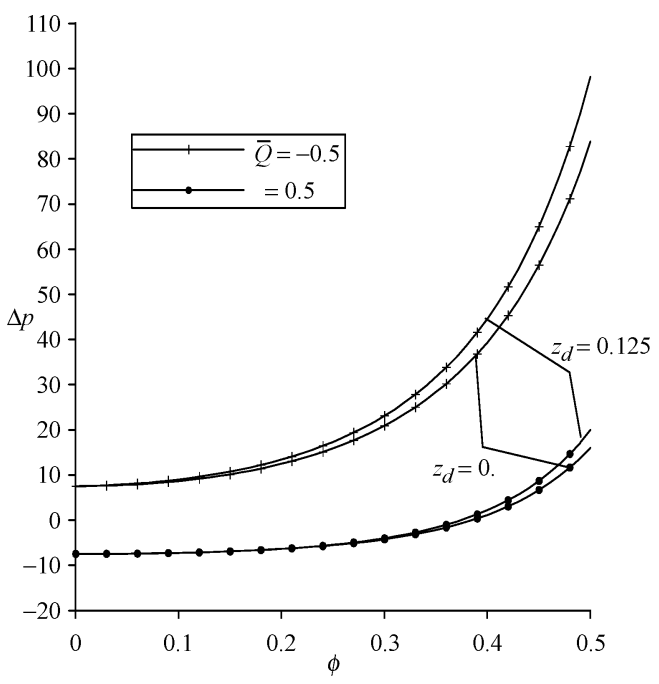

Figure 5. The variation of $\Delta p$ with $\phi$ for different values of $\bar{Q}$ and $z_{d}$ at $m=20, N=0.2, a=0.02, \delta=0.1$.

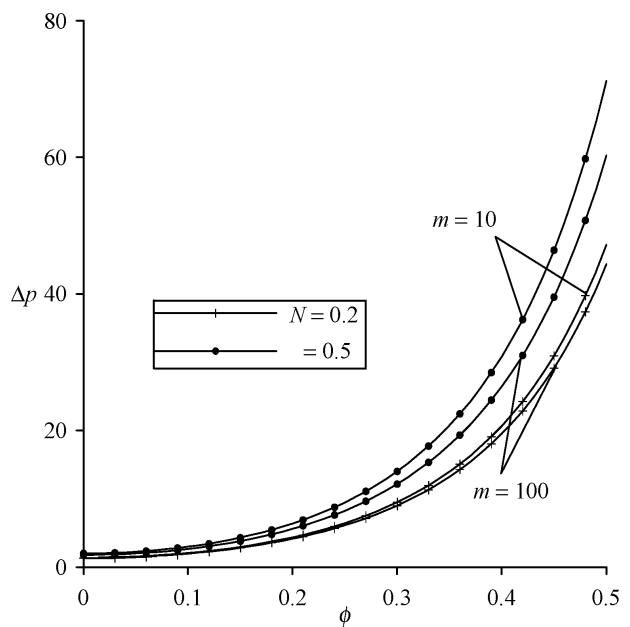

Figure 6. The variation of $\Delta p$ with $\phi$ for different values of $N$ and $m$ at $\bar{Q}=-0.1, z_{d}=0, a=0.01, \delta=0.005$.

outer friction force is greater than the inner friction force at the same values of the parameters.

\section{Streamlines and fluid trapping}

The phenomenon of trapping, whereby a bolus (defined as a volume of fluid bounded by a closed streamline in the wave frame) is transported at the wave speed. Figures 17 20 illustrates the streamline graphs for different values of the coupling number $N$ for a given fixed set of the other parameters. It is observed that the size of the trapping bolus increases as $N$ increases near the boundary wall (outer tube). The effects of micropolar parameter $m$ on the trapping with fixed values of the parameters are shown in Figures 2124. It is evident that the trapped bolus size increases with

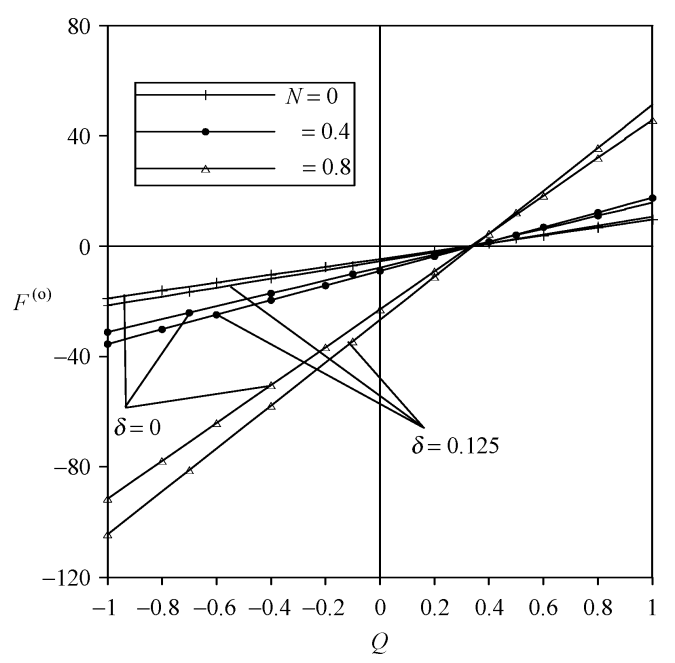

Figure 7. The variation of $F^{(o)}$ with $\bar{Q}$ for different values of $N$ and $\delta$ at $a=0.02, m=2, z_{d}=0, \phi=0.4$. 


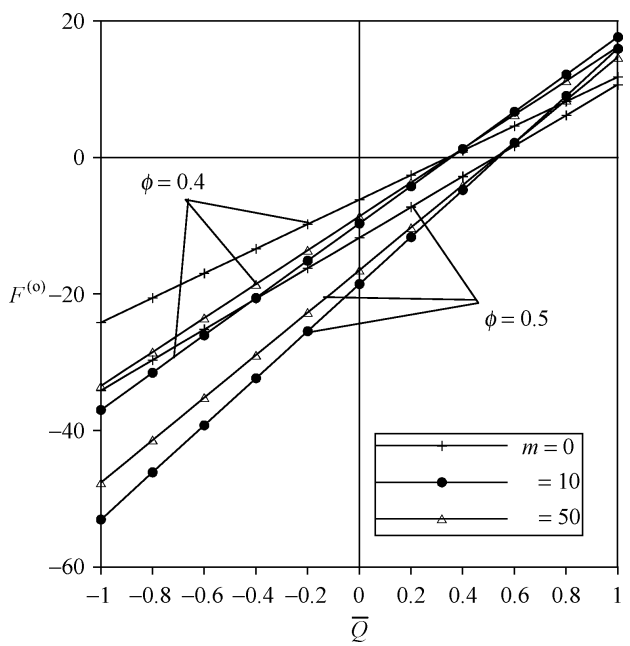

Figure 8. The variation of $F^{(o)}$ with $\bar{Q}$ for different values of $m$ and $\phi$ at $a=0.02, N=0.4, z_{d}=0, \delta=0.1$.

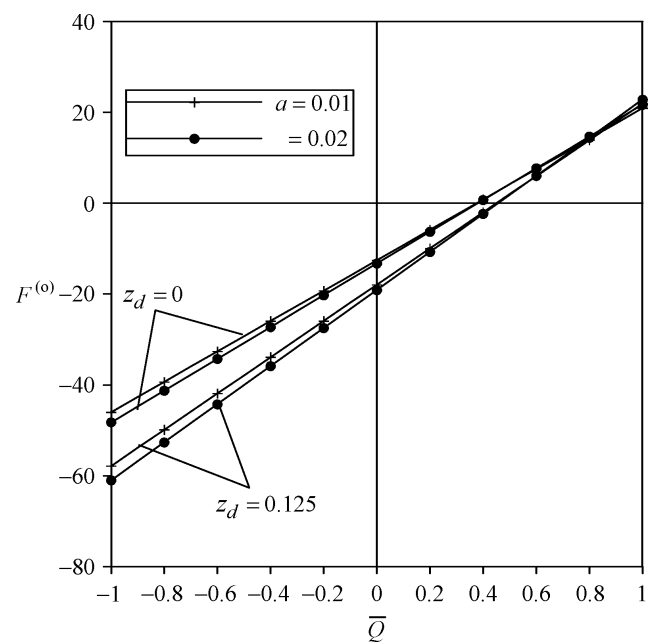

Figure 9. The variation of $F^{(o)}$ with $\bar{Q}$ for different values of $a$ and $z_{d}$ at $m=10, N=0.4, \phi=0.4, \delta=0.2$.

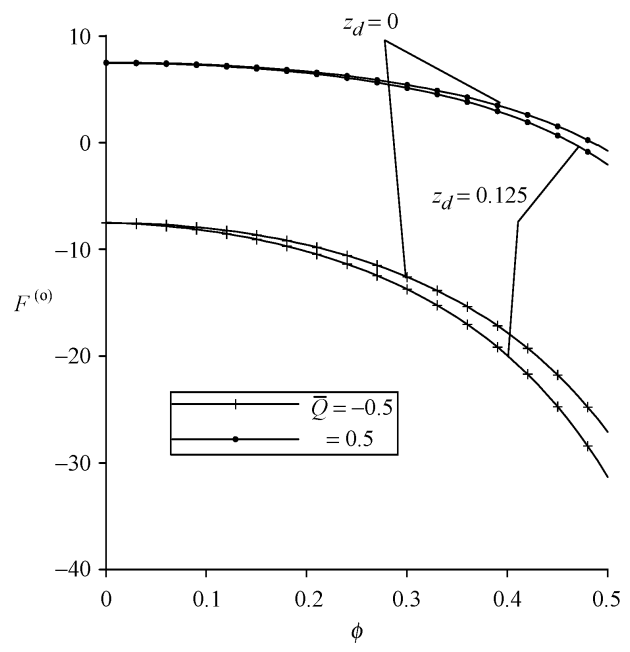

Figure 10. The variation of $F^{(o)}$ with $\phi$ for different values of $\bar{Q}$ and $z_{d}$ at $m=20, N=0.2, a=0.02, \delta=0.1$.

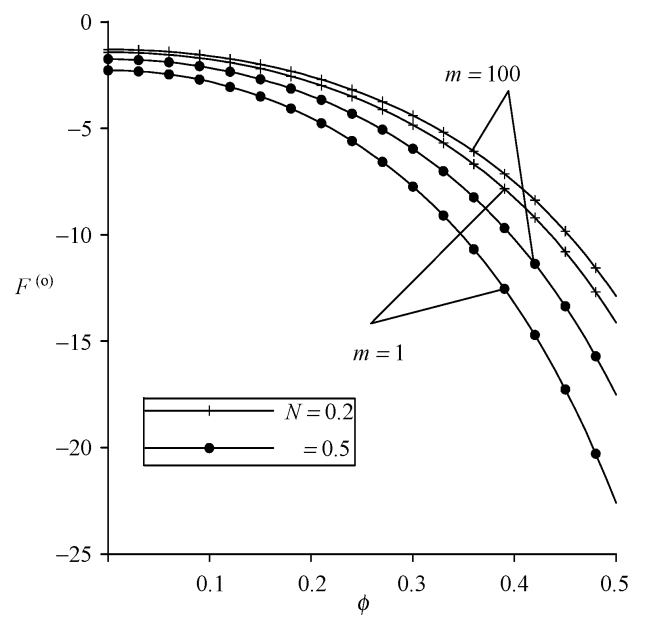

Figure 11. The variation of $F^{(o)}$ with $\phi$ for different values of $N$ and $m$ at $z_{d}=0, \bar{Q}=-0.1, a=0.01, \delta=0.05$.

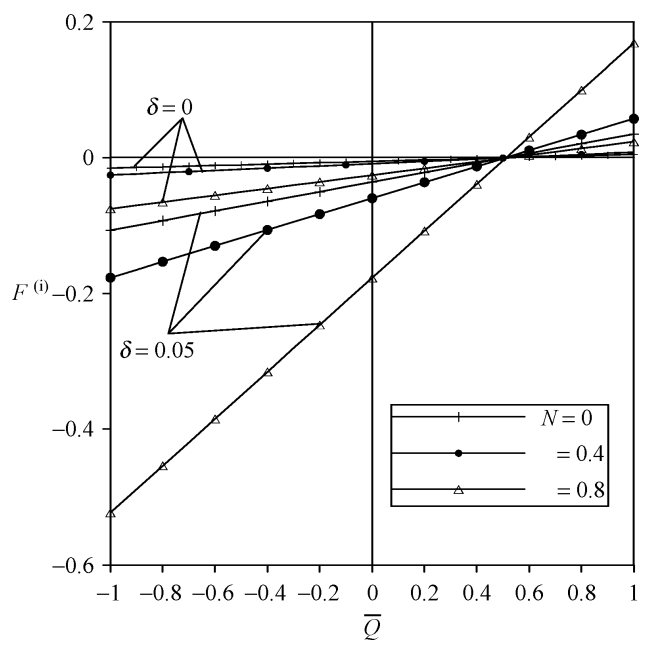

Figure 12. The variation of $F^{(i)}$ with $\bar{Q}$ for different values of $N$ and $\delta$ at $a=0.02, m=2, z_{d}=0, \phi=0.4$.

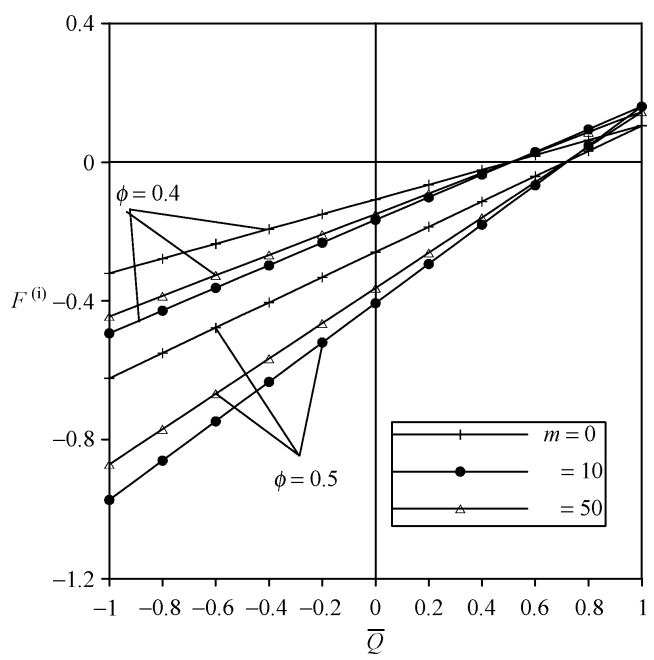

Figure 13. The variation of $F^{(i)}$ with $\bar{Q}$ for different values of $m$ and $\phi$ at $a=0.02, N=0.4, z_{d}=0, \delta=0.1$. 


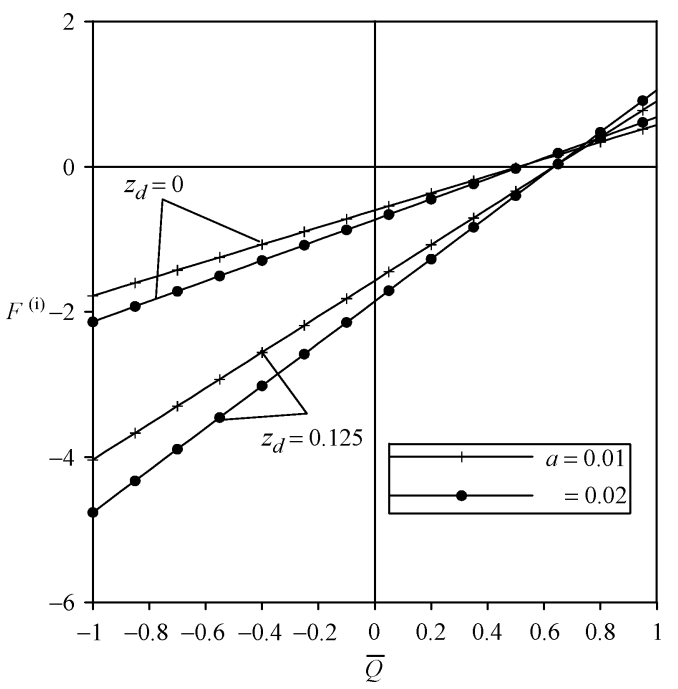

Figure 14. The variation of $F^{(i)}$ with $\bar{Q}$ for different values of $a$ and $z_{d}$ at $m=10, N=0.4, \phi=0.4, \delta=0.2$.

micropolar parameter increases $m$ up to a certain value of $m$ and decreases later. It is clear from Figures 25 and 26 that when the flow rate $\bar{Q}$ is small, there is no trapping bolus but by increasing the flow rate the trapping bolus appears and the number of the trapping bolus increases. The effects of the maximum height of the clot $\delta$ on the trapping are illustrated in Figures 27-29. It is evident that the size of trapping bolus decreases with increases $\delta$, with the other parameters being fixed.

\section{Concluding remarks}

We have presented a theoretical approach to study the annulus peristaltic flow where the inner tube has a clot on

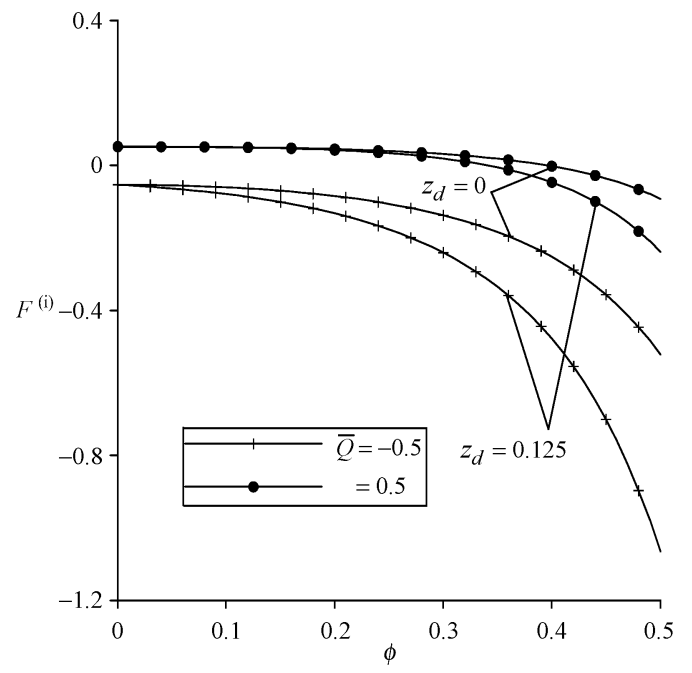

Figure 15. The variation of $F^{(i)}$ with $\phi$ for different values of $\bar{Q}$ and $z_{d}$ at $m=20, N=0.2, a=0.02, \delta=0.1$.

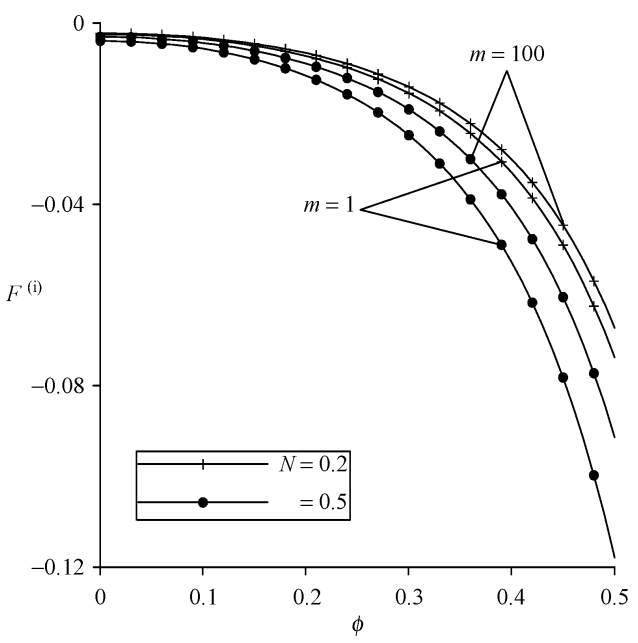

Figure 16. The variation of $F^{(i)}$ with $\phi$ for different values of $N$ and $m$ at $z_{d}=0, \bar{Q}=-0.1, a=0.01, \delta=0.05$.

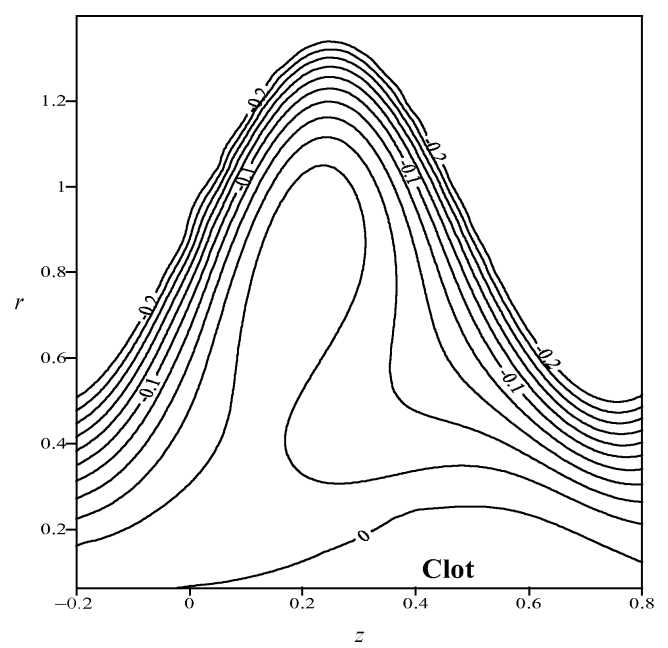

Figure 17. Graph of the streamlines for $N=0, m=2, \delta=0.25$, $a=0.02, \bar{Q}=0.5, \phi=0.4$.

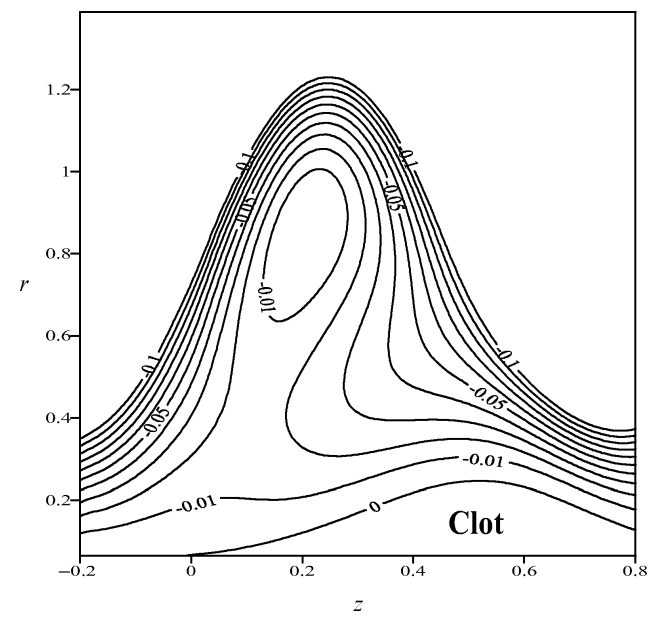

Figure 18. Graph of the streamlines for $N=0.3, m=2, \delta=$ $0.25, a=0.02, \bar{Q}=0.5, \phi=0.4$ 


$$
\frac{\Delta \Delta}{\Delta} \Delta
$$




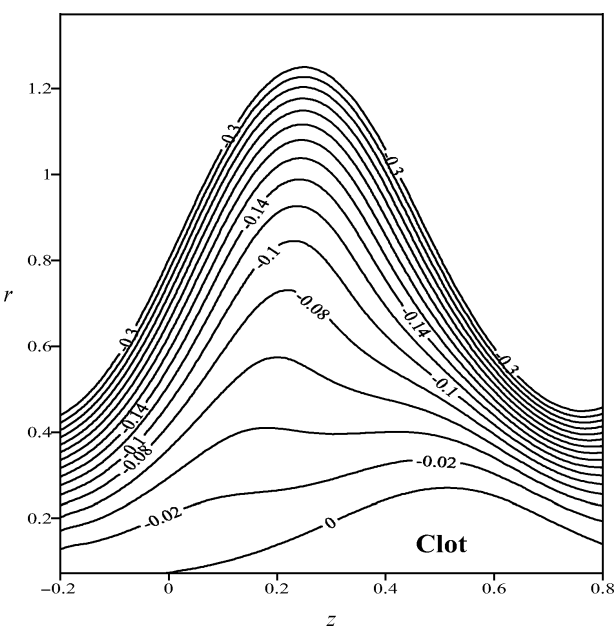

Figure 25. Graph of the streamlines for $\bar{Q}=0.1, N=0.8, m=$ $2, \delta=0.25, a=0.02, \phi=0.4$.

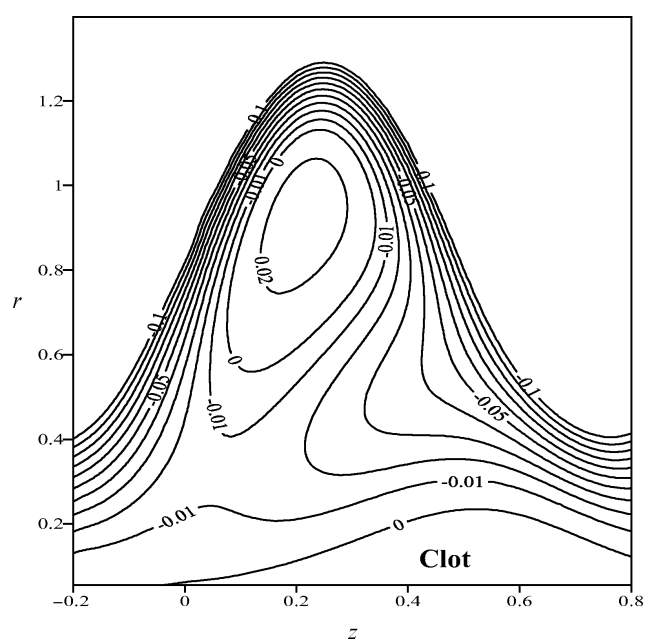

Figure 26. Graph of the streamlines for $\bar{Q}=0.6, N=0.8, m=$ $2, \delta=0.25, a=0.02, \phi=0.4$.

its wall. The present analysis can serve as a model that may help in understanding the mechanism of physiological (blood) flows in an annulus for a fluid behaving like a micropolar fluid. The main findings can be summarized as follows:

- The pressure rise increases when the maximum height attained by the clot increases.

- The pressure rise is higher in the case of a micropolar fluid model than that for a Newtonian fluid model.

- There is a linear relation between pressure rise and flow rate; an increase in the flow rate reduces the pressure rise and thus maximum flow rate is achieved at zero pressure rise and maximum pressure occurs at zero flow rate.

- The peristaltic pumping region becomes wider with the increase in the coupling number, the maximum

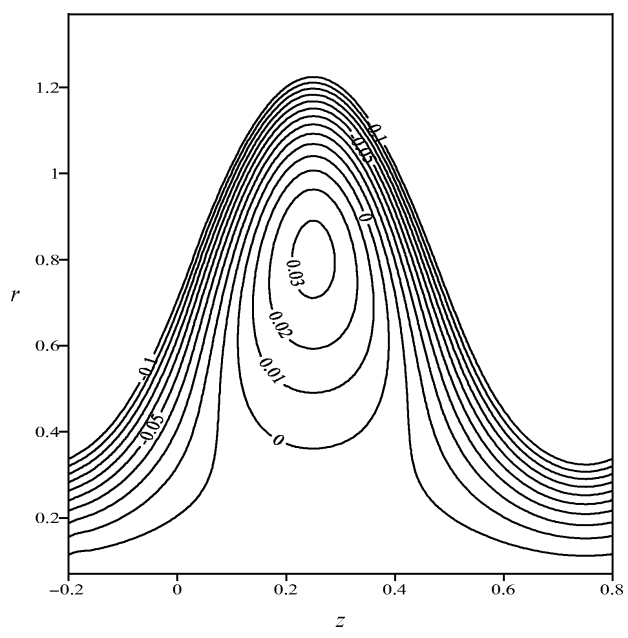

Figure 27. Graph of the streamlines for $\delta=0, N=0.8, m=2$, $\bar{Q}=0.5, a=0.02, \phi=0.4$.

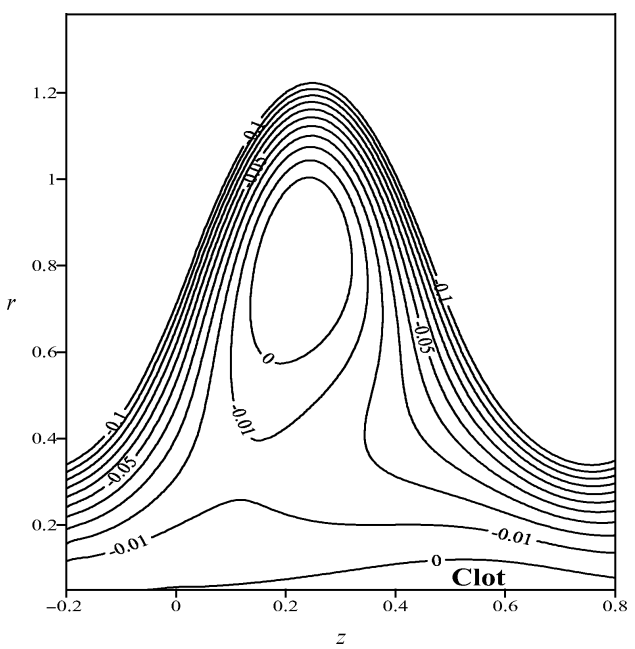

Figure 28. Graph of the streamlines for $\delta=0.1, N=0.8, m=$ $2, \bar{Q}=0.5, a=0.02, \phi=0.4$.

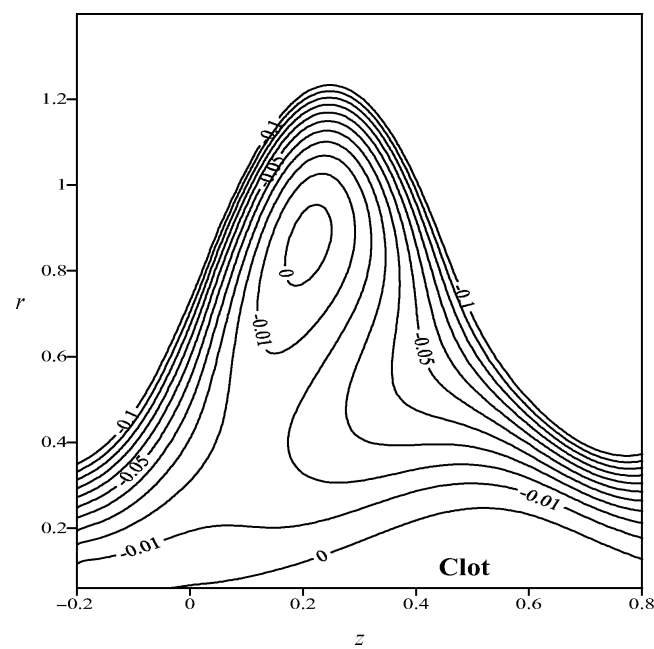

Figure 29. Graph of the streamlines for $\delta=0.25, N=0.8, m=$ $2, \bar{Q}=0.5, a=0.02, \phi=0.4$. 
height attained by the clot, amplitude ratio and the axial displacement of the clot.

- Elevating the values of the amplitude ratio produces more occlusion in the gap between the inner and outer tubes; this leads to increase in the pressure rise.

- The inner friction force behaves similar to the outer friction force for the same values of the parameters; moreover, the outer friction force is greater than the inner friction force for the same values of the parameters.

- The size of the trapping bolus increases as the coupling number increases near the boundary wall (outer tube), whereas it increases with increasing micropolar parameter (micropolar spin parameter effect) up to a certain value and then decreases for larger values.

- The size of trapping bolus decreases with increases in the height attained by the clot.

- In the absence of the inner tube (the clot model), the results are the same as those obtained by Srinivasacharya et al. (2003).

\section{References}

Cowin SC. 1968. Polar fluids. Phys Fluids 11:1919.

Eringen AC. 1966. Theory of micropolar fluids. J Math Mech. 16:1.

Hayat T, Mahomed FM, Asghar S. 2005. Peristaltic flow of a magnetohydrodynamic JohnsonSegalman fluid. Nonlinear Dyn. 40:375.

Jayaraman G, Sarkar A. 2005. Nonlinear analysis of arterial blood flow-steady streaming effect. Nonlinear Anal. 63:880.

Provost AM, Schwarz WH. 1994. A theoretical study of viscous effects in peristaltic pumping. J Fluid Mech. 279:177.

Rao AR, Mishra M. 2004. Peristaltic transport of a power-law fluid in a porous tube. J Non-Newtonian Fluid Mech. 121:163.

Shapiro AH, Jaffrin MY, Weinberg SL. 1969. Peristaltic pumping with long wavelengths at low Reynolds number. J Fluid Mech. 37:799.

Srinivasacharya D, Mishra M, Rao AR. 2003. Peristaltic pumping of a micropolar fluid in a tube. Acta Mech. 161:165.

Srivastava LM. 1986. Peristaltic transport of a couple stress fluid. Rheol Acta. 25:638.

Srivastava LM, Srivastava VP. 1988. Peristaltic transport of powerlaw fluid: application to the ductus of efferuntus of the reproductive tract. Rheol Acta 27:428.

Vajravelu K, Sreenadh S, Ramesh Babu V. 2005. Peristaltic transport of a Herschel-Bulkley fluid in an inclined tube. Int $\mathrm{J}$ Non-Linear Mech. 40:83.

\section{Appendix}

$$
\begin{aligned}
& a_{11}=r_{2} I_{1}\left(m r_{2}\right)-r_{1} I_{1}\left(m r_{1}\right), \\
& a_{12}=I_{1}\left(m r_{2}\right) K_{1}\left(m r_{1}\right)-I_{1}\left(m r_{1}\right) K_{1}\left(m r_{2}\right), \\
& a_{13}=I_{0}\left(m r_{1}\right)-I_{0}\left(m r_{2}\right) \\
& a_{14}=r_{1} K_{1}\left(m r_{1}\right)-r_{2} K_{1}\left(m r_{2}\right), \\
& a_{15}=K_{0}\left(m r_{1}\right)-K_{0}\left(m r_{2}\right),
\end{aligned}
$$

$$
\begin{aligned}
& a_{16}=r_{2}^{2}-r_{1}^{2}, A=\frac{1-N}{2-N}, F=\frac{N}{m} \\
& a_{17}=r_{1} I_{1}\left(m r_{2}\right)-r_{2} I_{1}\left(m r_{1}\right), \\
& a_{18}=r_{1} K_{1}\left(m r_{2}\right)-r_{2} K_{1}\left(m r_{1}\right) \text {, } \\
& a_{19}=r_{2} K_{1}\left(m r_{1}\right)+r_{1} K_{1}\left(m r_{2}\right), \\
& a_{21}=I_{0}\left(m r_{1}\right) K_{0}\left(m r_{2}\right)-I_{0}\left(m r_{2}\right) K_{0}\left(m r_{1}\right), \\
& a_{22}=\ln r_{1} I_{0}\left(m r_{2}\right)-\ln r_{2} I_{0}\left(m r_{1}\right), \\
& a_{23}=a_{11} a_{15}-a_{13} a_{14}, \\
& a_{24}=2 F a_{23}-4 a_{12} r_{1} r_{2} \ln \left(\frac{r_{1}}{r_{2}}\right), \\
& a_{25}=\ln r_{1} K_{0}\left(m r_{2}\right)-\ln r_{2} K_{0}\left(m r_{1}\right), \\
& a_{26}=a_{14} I_{0}\left(m r_{1}\right)-a_{11} K_{0}\left(m r_{1}\right) \text {, } \\
& a_{27}=a_{11} K_{0}\left(m r_{2}\right)-a_{14} I_{0}\left(m r_{2}\right), \\
& a_{28}=r_{1}^{3} K_{1}\left(m r_{1}\right)+r_{2}^{3} K_{1}\left(m r_{2}\right) \text {, } \\
& a_{29}=F a_{13}-a_{11}, a_{32}=1-a_{16} a_{12} \text {, } \\
& a_{31}=\frac{1}{F A}\left[a_{17} a_{15}-a_{13} a_{18}\right] \text {, } \\
& a_{33}=F a_{16} a_{15}-a_{28}+r_{1} r_{2}\left(a_{19}+2 a_{18} \ln \left(\frac{r_{2}}{r_{1}}\right)\right) \text {, } \\
& a_{35}=\frac{1}{F}\left[2 a_{12} r_{1} r_{2}\right], \\
& a_{34}=F a_{16} a_{21}+2 r_{1} r_{2} a_{18} a_{22}+a_{31}+a_{38}, \\
& a_{36}=a_{16} a_{29} \text {, } \\
& a_{37}=2 r_{1} r_{2} a_{17} \ln \left(\frac{r_{2}}{r_{1}}\right), \\
& a_{38}=2 r_{1} r_{2} a_{17} a_{25} \text {, } \\
& a_{39}=2 F\left(a_{23}+a_{35} \ln \left(\frac{r_{1}}{r_{2}}\right)\right), \\
& a_{41}=a_{36}+a_{37}, \\
& a_{43}=2 a_{18} r_{1} r_{2} \ln \left(\frac{r_{1}}{r_{2}}\right)-a_{16}\left(F a_{15}+a_{14}\right), \\
& a_{44}=r_{2} I_{0}\left(m r_{1}\right) K_{1}\left(m r_{2}\right) \text {, } \\
& a_{45}=r_{1} I_{0}\left(m r_{2}\right) K_{1}\left(m r_{1}\right) \text {, } \\
& a_{46}=F r_{1} I_{1}\left(m r_{1}\right) K_{0}\left(m r_{2}\right) \text {, } \\
& a_{47}=2 r_{2} r_{1} I_{1}\left(m r_{1}\right) \ln \left(\frac{r_{1}}{r_{2}}\right), \\
& a_{48}=a_{26}+a_{35} \ln r_{1}, \\
& a_{49}=a_{27}+a_{35} \ln r_{2}, \\
& a_{51}=I_{1}\left(m r_{1}\right)+I_{0}\left(m r_{2}\right), \\
& a_{52}=I_{0}\left(m r_{1}\right) K_{1}\left(m r_{2}\right)+I_{1}\left(m r_{2}\right) K_{0}\left(m r_{1}\right), \\
& a_{53}=2 F-\frac{m}{I_{1}\left(m r_{1}\right)}\left(a_{51} a_{46}+F a_{52} r_{2} I_{1}\left(m r_{1}\right)+a_{12} a_{47}\right) \text {, }
\end{aligned}
$$




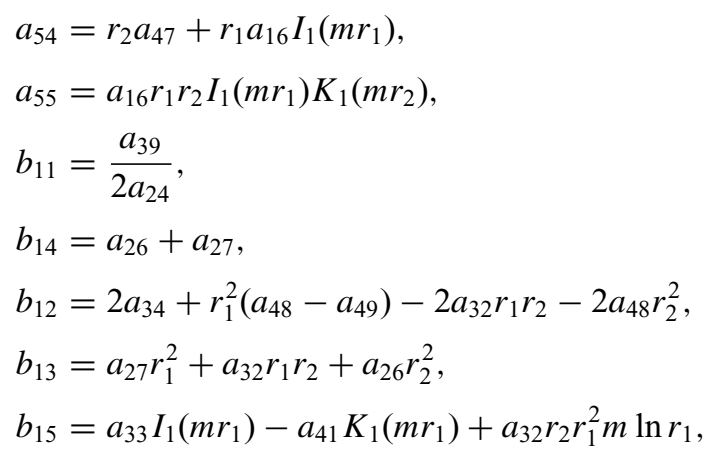

$$
\begin{aligned}
b_{16} & =r_{2}^{2} \ln r_{2}-r_{1}^{2} \ln r_{1}, \\
b_{17} & =a_{33} a_{11}+a_{41} a_{14}, \\
b_{18} & =a_{48}-a_{49}, \\
b_{19} & =\frac{2 m a_{24}}{A F}, \\
\xi & =\frac{2 m a_{16} a_{39}}{A F}, \\
\eta & =4\left(b_{17}+a_{32} b_{16} m r_{1} r_{2}\right)-m a_{42}\left(2 a_{34}-b_{18} a_{16}\right)
\end{aligned}
$$



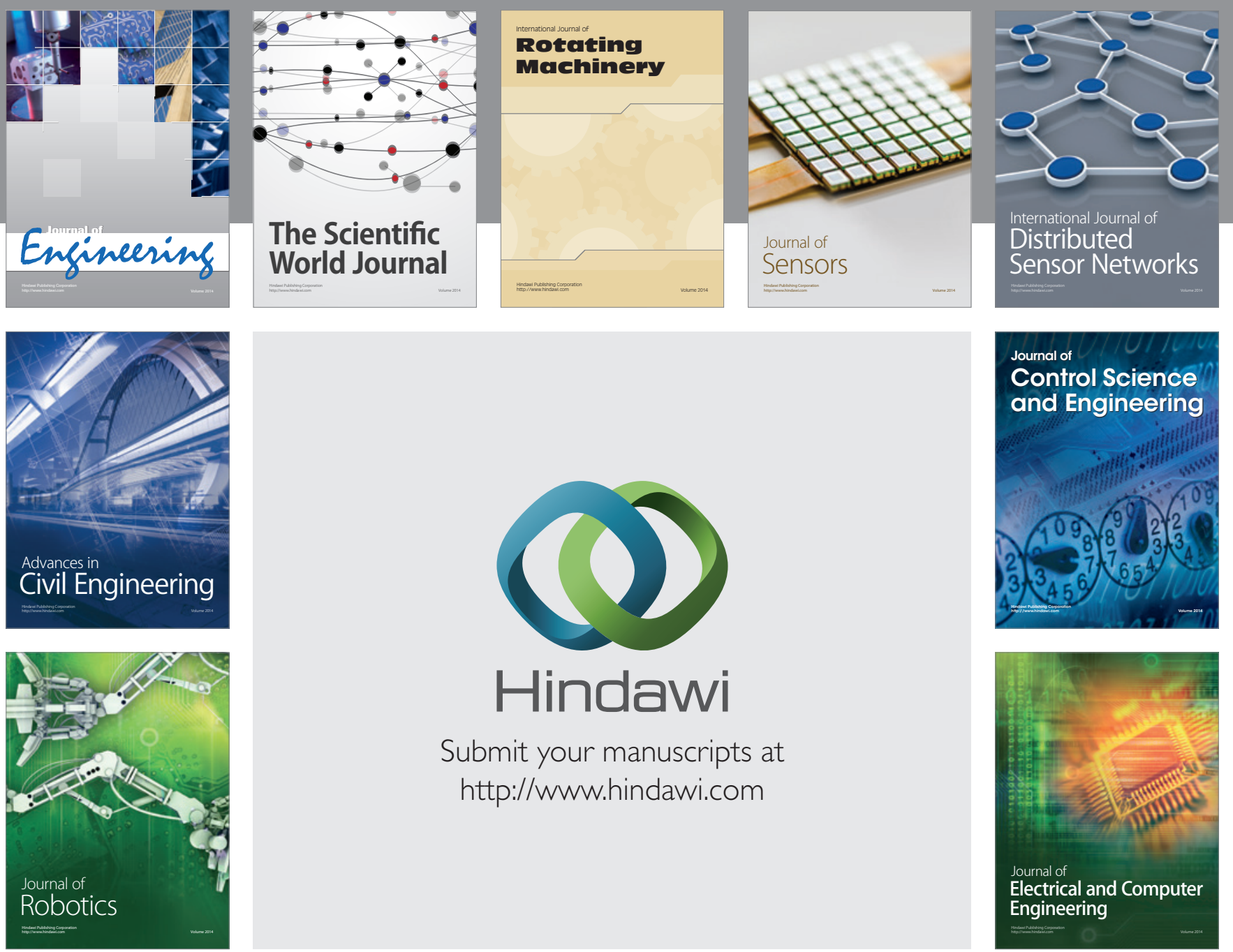

Submit your manuscripts at

http://www.hindawi.com
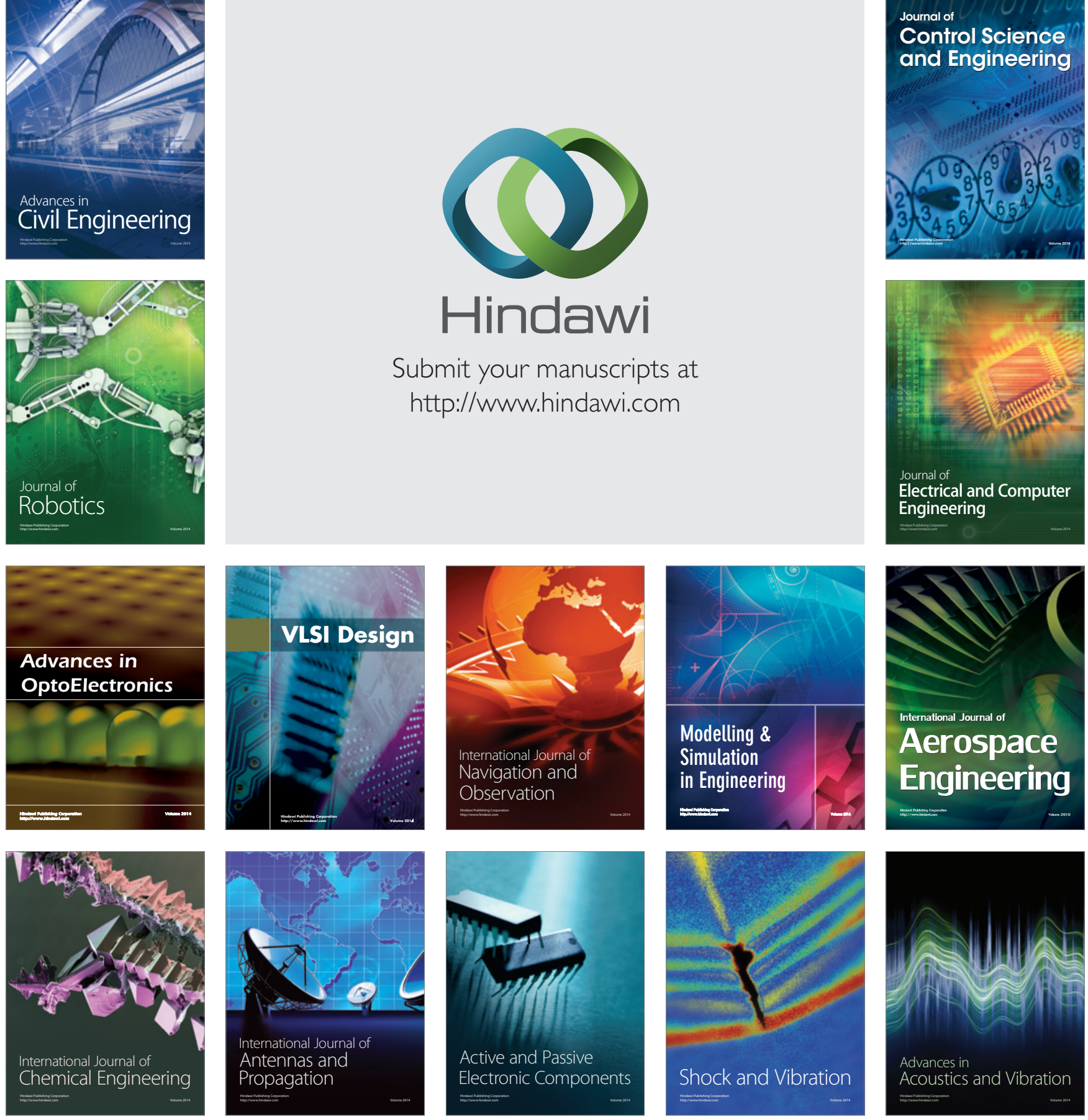